\title{
LAS VENTAJAS DEL IoT EN EL DISEÑO DE LABORATORIOS REMOTOS: MYGATEWAY
}

\author{
Marco Antonio Márquez Sánchez \\ Universidad de Huelva marcoa@pi.uhu.es \\ María Reyes Sánchez Herrera \\ Universidad de Huelva reyes.sanchez@die.uhu.es \\ Andrés Mejías Borrero \\ Universidad de Huelva mjias@uhu.es \\ José Manuel Andújar Márquez \\ Universidad de Huelva andujar@uhu.es
}

\begin{abstract}
Resumen
En la época del internet de las cosas se ha vuelto relativamente simple controlar desde cualquier localización, a través de nuestros dispositivos smart, cualquier electrodoméstico de nuestra vivienda, o la iluminación o el acondicionamiento de aire, entre otros. Aunque con reservas, esa revolución está llegando también a las industrias. Sin embargo, en el ámbito de la educación, todavía parece realmente complicado hacer accesibles desde internet los laboratorios, a pesar de las ventajas que esto reportaría. Para superar esas dificultades, se presenta en este artículo un dispositivo que permite integrar como servicio en la nube cualquier experiencia didáctica o de investigación, sea de la naturaleza que sea y que use cualquier protocolo de comunicaciones entre los distintos dispositivos que la componen. Su instalación y configuración son simples e intuitivas, por lo que la persona que lo ponga en marcha no tendrá que ser especialista en comunicaciones. El dispositivo es parte de un sistema mayor denominado ENLARGE.
\end{abstract}

Palabras clave: Educación en ingeniería, laboratorios remotos, el internet de las cosas, comunicaciones, nube.

\section{INTRODUCCIÓN}

El internet de las cosas (internet of things, IoT), [1], está revolucionando nuestra rutina diaria en muchos de sus ámbitos, muchos de nosotros usamos smartwatch, la mayoría tenemos smartphone y cada vez está más extendido el uso de smartDevices (acceso a camaras, control de electrodomésticos, smart lavadoras, frigorificos, aire acodicionado, domótica doméstica, wifi smart plug, wifi smart switch, wifi smart lamp, asistentes de voz como
Alexa o Google Home), entre otros. Una gran parte del éxito del IoT radica en la facilidad de interconectar cosas entre si (comunicaciones M2M, machine to machine) y facilitar la interacción de las personas con estos dispositivos (comunicaciones M2P, machine to person), que además se puede hacer desde los dispositivos smart: el reloj, el teléfono móvil, la tablet, la televisión además de cualquier ordenador con conexión a la red.

Con respecto al ámbito industrial, aunque muchas empresas ya se benefician de las ventajas de internet en su organización a nivel IT (industrial technology), son muy pocas las que han incorporado esas ventajas a su capa OT (operation technology). Téngase en cuenta que gestionar desde internet la capa OT implica configurar y acceder en remoto a sensores, actuadores y controladores. Y es ahí donde podría entrar de lleno el IoT. Sin embargo, esta revolución presenta riesgos inherentes, [2], como por ejemeplo el hecho de que los datos personales del usuario, de sus actividades lúdicas e incluso de configuración de sus electrodomésticos estén disponibles en servidores desconocidos y a disposición de todo el que quiera conseguirlos. Esto dificulta la adopción del IoT por las industrias. Actualmente se barajan soluciones a estos problemas que se integran dentro del movimiento Industria 4.0 o el IoT industrial (industrial IoT, IIoT), [3].

Por otro lado, cada día toma más protagonismo la nube y los servicios integrados en la misma, [4]. Esto constituye un paso más en la integración de los servicios en red. Son numerosas las ventajas adquiridas por las empresas que tienen su capa IT integrada en la nube. Y la mejora puede ser mucho mayor con la convergencia de las dos capas (IT y OT). 
Sin embargo, la integración de la capa OT en la nube no es algo trivial y está tendiendo a resolverse de forma específica por cada empresa en cada industria en función de los protocolos usados y los equipos físicos que tengan en funcionamiento.

Estas soluciones específicas recuerdan en gran medida a lo que se ha venido haciendo con los laboratorios remotos en las últimas décadas: el acceso remoto a una experiencia de laboratorio se desarrollaba para darle solución a ese caso concreto sin que se tuviera en cuenta su extrapolabilidad a otras posibles situaciones, [5]-[8]. Además, por lo general, el problema de las comunicaciones es uno de los más complejos con el que se encuentra el desarrollador de laboratorios, en contraposición con la amigabilidad proporcionada por el IoT. Y, sin embargo, la seguridad en el acceso es tan importante en los laboratorios como en el campo industrial.

Lo que se presenta en este artículo es un dispositivo (myGateway de aquí en adelante) que permite integrar como servicio en la nube cualquier experiencia didáctica o de investigación, sea de la naturaleza que sea y que use cualquier protocolo de comunicaciones entre los distintos dispositivos que la componen. Los sensores y actuadores estarán controlados de manera digital desde un microcontrolador. El dispositivo que se presenta ofertará sus servicios en la nube simplemente conectándolo a la misma red interna (Local Area Network, LAN) en la que esté conectado ese controlador sin necesidad de disponer de direcciones válidas en internet. $\mathrm{Y}$ las necesidades de configuración de myGateway son mínimas.

Además, en este artículo se describe el procedimiento $\mathrm{y}$ las herramientas propuestas para integrar como servicio en la nube una experiencia. Aunque myGateway se puede usar también con experiencias controladas mediante LabView, [9], o Matlab, [10], el artículo se centra en la implementación a partir de plataformas abiertas de hardware y software libre (Arduino, [11], en concreto, aunque también podrían ser las tarjetas Phidgets, [12], Orange Pi, [13], etc). $\mathrm{Al}$ presentar las distintas herramientas necesarias, se irán introduciendo pequeños ejemplos que, en conjunto, nos llevarán al desarrollo de un caso de uso sencillo. Estos ejemplos se han incorporado para aclarar la forma de usar las distintas herramientas y para mostrar la facilidad de integración de myGateway. Sin embargo, los ejemplos no muestran su potencialidad, ya que myGateway puede gestionar múltiples microcontroladores al mismo tiempo.

Así, el resto del artículo se organiza de la siguiente forma: en la sección 2 se describe el funcionamiento de myGateway como parte de un sistema más amplio. En la sección 3 se presenta la herramienta de administración propuesta para myGateway. En la sección 4 se describe brevemente la herramienta a usar para configurar la aplicación de control de la experiencia propuesta $\mathrm{y}$ en la sección 5 la correspondiente al desarrollo de la aplicación con la que se accederá a la experiencia desde internet. Finalmente, en la sección 6 se presentan las conclusiones más relevantes.

\section{EL FUNCIONAMIENTO DE myGATEWAY}

myGateway es parte de un sistema mayor denominado ENLARGE (rEmote coNtroLled Access to inteRnet of thinGs dEvices) que oferta como servicio en la nube el acceso a una instalación física (IF). Esa IF puede ser una planta experimental de laboratorio docente o de investigación, una planta industrial o un conjunto de dispositivos domésticos. myGateway, así como los distintos módulos de ENLARGE, su forma de adquisión y sus funciones de uso se pueden consutar en la ULR (uniform resource locator) http://enlargeirs.dia.uned.es.

A continuación se describe brevemente la constitución y el funcionamiento de myGateway.

Físicamente, myGateway es una placa base simple (single-board computer SBC), que puede ser de la familia i86 o ARM, con el sistema operativo Linux. En el caso de que la IF cuente ya con una aplicación de control desarrollada en aplicaciones propietarias como LabView o Matlab, existen componentes para que myGateway se integre directamente entre esa aplicación y la LAN en la que está conectada.

Pero myGateway también se puede usar en plataformas abiertas con software libre, figura 1. Para ello, en el mismo dispositivo se ejecuta la aplicación EJS (Easy Java Simulation, [14]). Esta aplicación es una herramienta de creación gratuita escrita en Java, que facilita las simulaciones interactivas. EJS cuenta además con los elementos necesarios para acceder a plataformas abiertas de hardware. En estos casos, myGateway se conectará a los microcontroladores Arduino elegidos para controlar la IF.

De acuerdo con la figura 1, la conexión con el microcontrolador se hará mediante USB (universal serial bus) y EJS ejercerá su control mediante el elemento Arduino (en el caso que nos ocupa). Arduino será el que interactue con la IF. Asimismo, el elemento arduinoWebsocket es el encargado de encapsular las instrucciones de entrada/salida del microcontrolador bajo el protocolo websocket. De esta forma, es posible interactuar sobre la IF desde el navegador. Los elementos Arduino y arduinoWebsocket no necesitan configuración. 
Con una de las opciones descritas (LabView, Matlab y EJS), ya temenos la conexión entre myGateway y la IF, (1) en la figura 2. El siguiente paso es implementar en una nube el acceso a esa IF. Para eso, se necesitan dos servicios complementarios. Uno de ellos, denominado myPublisher, se ejecuta en myGateway y es el que se encarga de solicitar la suscripción del acceso en la nube. El otro, denominado myVirtualFrontier, se ejecuta en un servidor accesible desde internet (con IP válida) y se encarga de publicar las subcripciones de acceso. La acción de estas dos aplicaciones consituye la conexión (2) de la figura 2.

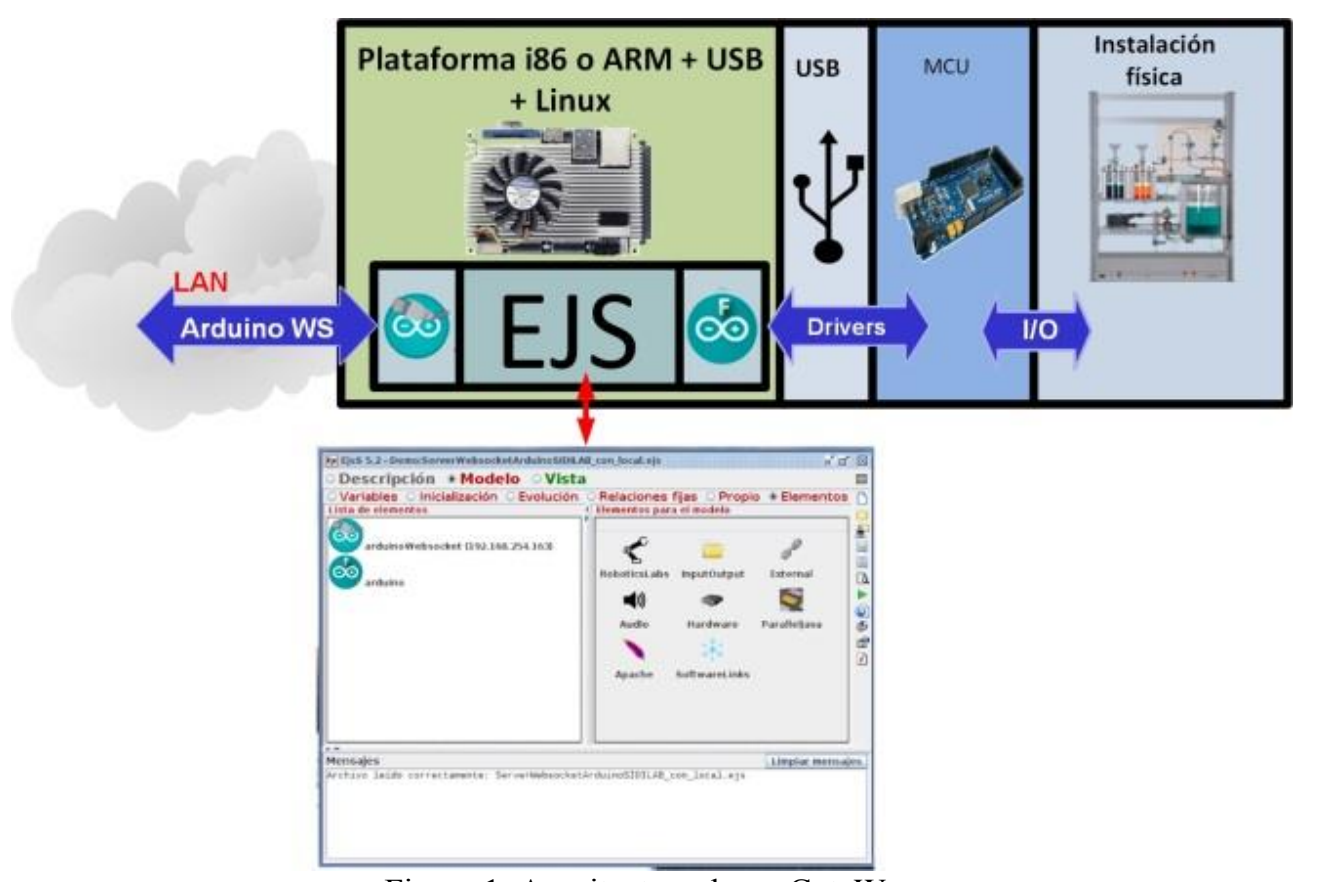

Figura 1. Arquitectura de myGateWay

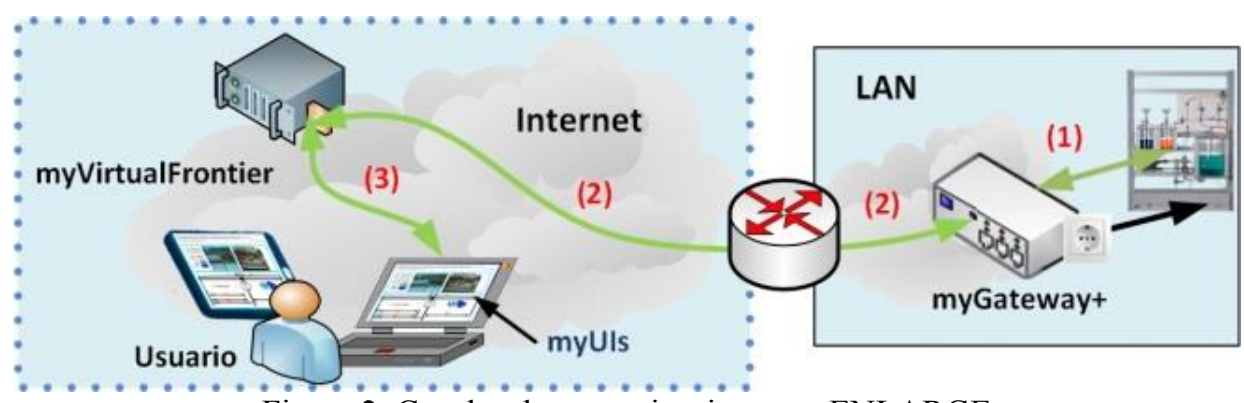

Figura 2. Canales de comunicaciones en ENLARGE

De esta forma, el usuario podrá solicitar el acceso a la IF mediante la descarga de una página web que utiliza JavaScript para conectar con el elemento arduinoWebsocket, que está conectoado con la IF a traves del elemento arduino, conexión (3) en la figura 2. Esa página web se denomina en este artículo myUI (UI, inerfaz de usuario en inglés) y estará alojada en un servidor de aplicaciones instalado en myGateway. Ese servidor de aplicaciones se usará además para controlar el acceso a la IF.

Además, y especialmente conveniente en el caso de las experiencias de laboratorio, myUI puede ser integrada en un gestor de aprendizaje (LMS learning management system en inglés) mediante el protocolo
LTI (lower tactical internet). Además, el módulo EJSApp, desarrollado por la UNED (Universidad Nacional de Educación a Distancia), [15], permite alojar myUI en un servidor moodle y controlar los accesos mediante un gestor de reserva.

myGateway contiene un servidor de video que permite administrar el flujo proporcionado por una cámara USB directamte conectada a un puerto de myGateway. Ademas dispone de la posibilidad de integrar myPDU (power distributor unit), que permite conectar la alimentación necesaria de los componentes de la IF al solicitar el acceso y desconectarla al salir. Con estos dos elementos, myGateway cuenta con todas las funciones 
necesarias para hacer accesible desde internet cualquier IF.

\section{HERRAMIENTA DE GESTIÓN DE ENLARGE}

Todos los módulos de ENLARGE se gestionan mediante la misma aplicación, que se denomina myAdministration, figura 3. myAdministration tiene dos niveles de uso: el del administrador y el de usuario (opcion que se denomina myFrame). El nivel de myFrame es el que se muestra en la figura 3. En la esquina inferior izquierda se encuentra un botón para cambiar de un nivel a otro. Desde myFrame, el usuario autorizado por el administrador, puede descargarse myUI. Esta es por tanto la tercera opción (después de la descarga directa de la página web y el acceso mediante LSM) para materializar la conexión (3) de la figura 2. En este caso, el usuario se identifica al entrar en la aplicación myAdministration.

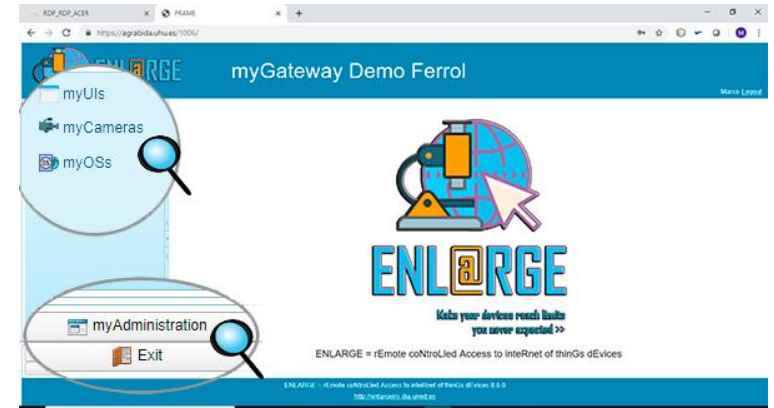

Figura 3. Opción myFrame de myAdministration.

Además, desde myFrame, el usuario que cuente con los permisos necesarios puede también acceder a los flujos de video de las cámaras situadas en la IF y al sistema operativo de myGateway y de cualquier dipositivo conectado a la misma red que permita los protocolos RDP (remote desktop protocol), VNC (virtual network computing), SSH (secure shell) y Telnet (telecommunication network).

El nivel de administración, cuyo marco es myAdministration, ofrece muchas más opciones que las disponibles en myFrame, figura 4 , y cada una de ellas son de gestión y configuración y no solo de acceso como en myFrame.

Entre todas esas opciones, se encuentra la que configura myGateway con los correspondientes parámetros o la que importa los ficheros de definición de myUI que se generan desde EJSS (Easy Java/JavaScript Simulations), desplegada en la figura 4. Estos ejecutables se suben comprimidos en .zip y su desarrollo se explica en la sección 5.
Desde "Administración de myUIs", el administrador de myGateway sube el fichero .zip que genera EJSS correspondiente a cada myUI, lo instala en el sistema y le da permisos a los usuarios que considere oportuno para que se la puedan descargar desde el nivel myFrame de la aplicación.

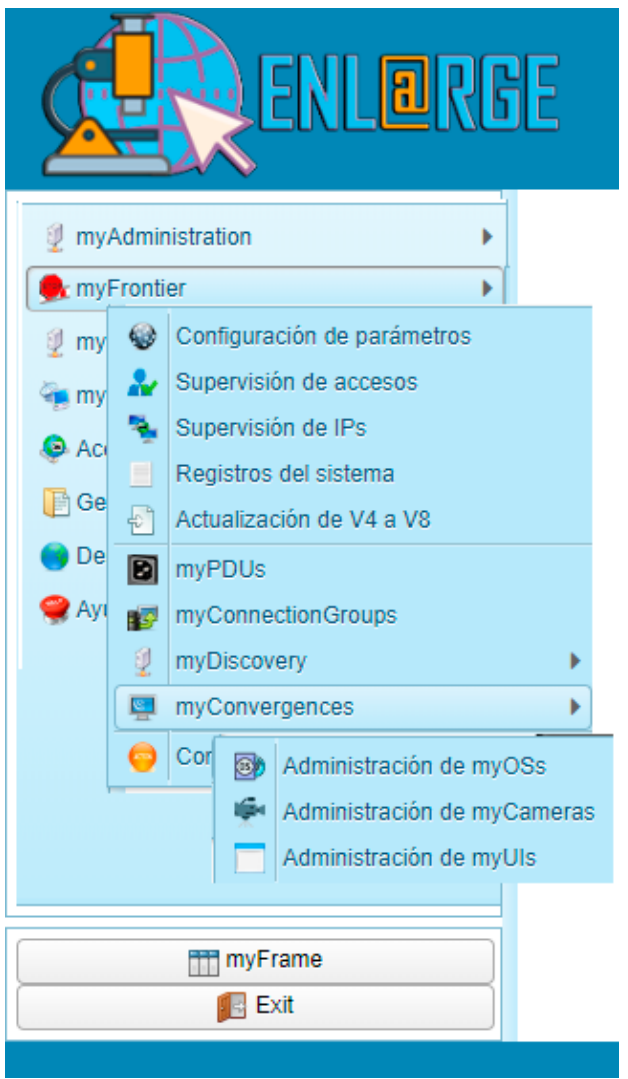

Figura 4. Nivel de administración de myAdministration.

\section{EL DESARROLLO DEL PROGRAMA DE CONTROL}

Como se ha indicado en la sección 2, el sistema operativo en myGateway es Linux y tiene EJS instalado. Para desarrollar el sistema de control de la IF es necesario acceder a esa aplicación en myGateway. myAdministration ofrece la opción de esa conexión desde el nivel myFrame (myOSs). Seleccionando esa opción, los usuario autorizados acceden a la aplicación EJS que se está ejecutando en myGateway a traves del navegador, figura 5. Ahí se puede desarrollar la aplicación de control de la IF.

Si los requerimientos de la IF se limitan a controlar desde el navegador las acciones de entrada y salida, el sistema queda configurado definiendo únicamente el modo de los pines del microcontrolador en la sección "Inicialización" del programa en EJS. A continuación se analizan las órdenes de la parte inicialización en un ejemplo sencillo: 
arduino.connect(arduino.list()[0], 57600); arduino.pinMode(2, arduino.INPUT); arduino.pinMode(3, arduino.PWM); arduino.pinMode(4, arduino.OUTPUT); arduino.pinMode(9, arduino.SERVO); arduino.digitalWrite $(4,1)$; arduino.servoWrite $(9,0)$; arduinoWebsocket.setArduino(arduino); arduinoWebsocket.connect();

La primera linea, inicializa la linea serie de la interfaz USB del microprocesador. Las lineas 2, 3, 4 y 5 configuran los pines en modo entrada, pwm, salida y servo. La linea 6 pone a 1 la salida 4 , la línea 7 pone a $0^{\circ}$ el servo.

Si las necesidades de control de la IF son mayores y por tanto el programa más complejo, se puede implementar un controlador con el programa necesario con ayuda de las opciones de EJS e incluyendo algoritmos en Java. En estos casos más complejos, las interacciones sobre la IF demandadas desde el navegador del usuario son recogidas en el programa de control de EJS y no en los pines del microcontrolador.

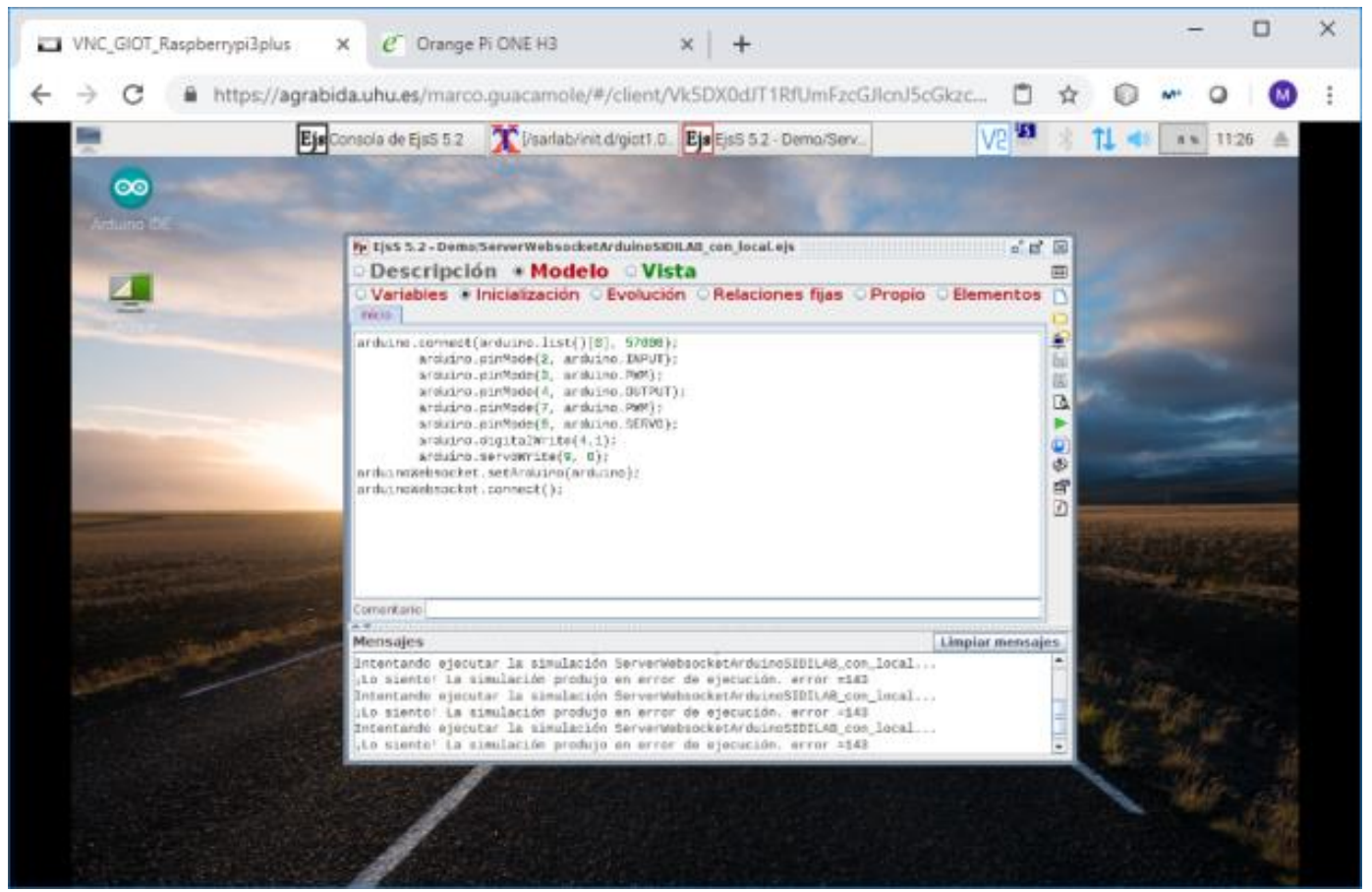

Figura 5. Vista del navegador de myGateway al que se accede desde myOSs en myAdministration.

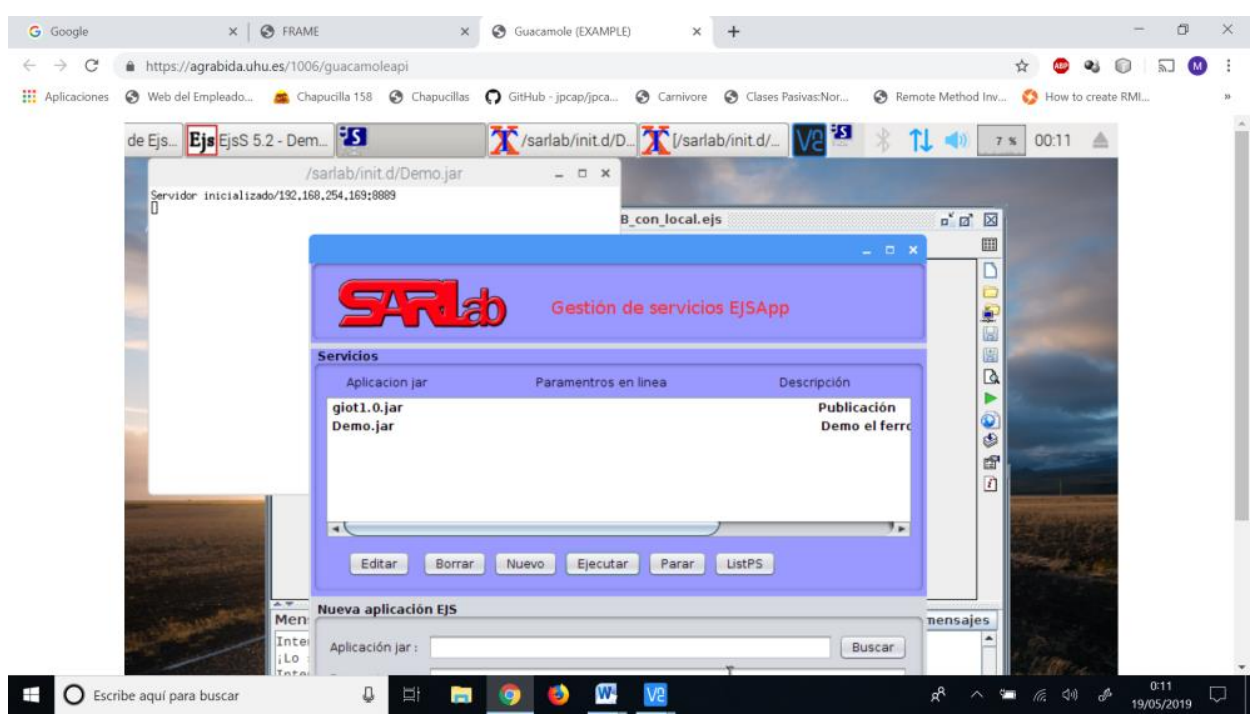

Figura 6. Inicialización automática del programa de control. 
Asimismo, sería conveniente en los casos complejos, interpretar las interacciones desmandadas por el usuario desde el navegador antes de realizarlas sobre el microcontrolador por si es necesaio intervenir con medidas de seguridad sobre los actuadores de la IF. Ademas, para usos avanzados, EJS en myGateway incorpora elementos Modbus que permiten interactuar con componentes industriales presentes en IF complejas.

Una vez depurado el programa, EJS permite compilarlo en un fichero jar. Y mediante el gestor de aplicaciones EJS instalado en myGateway podemos hacer que esta aplicación se inicie automaticamente al iniciar myGateway, figura 6 .

\section{LA IMPLEMENTACIÓN DE myUI}

La herramienta propuesta para implementar myUI es EJSS, que contiene dos elementos que debe incorporar myUI:

- El elemento ENLARGE, que establece los canales de comunicación entre myUI (que se ejecuta en el dispositivo del usuario, ordenador personal, tablet o smartphone) y la IF.

- El elemento ArduinoWeb que permite controlar desde myUI las órdenes de entrada/salida del microprocesador (Arduino en este caso) que interactua con la IF. myUI contendrá tantos elementos ArduinoWeb como placas Arduino haya en la IF.

El primer paso para crear myUI es incluir los dos elementos (ENLARGE y arduinoWeb) en la sección Elementos del Modelo de EJSS, figura 7.

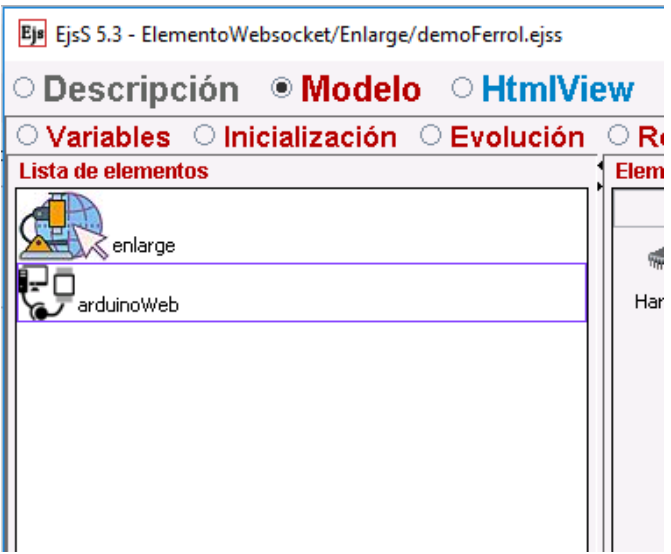

Figura 7. Elementos de EJSS necesarios en myUI.

ArduinoWeb no necesita configuración. La configuración de ENLANGE consiste en informar los siguientes parámetros:

- El nombre y el puerto del servidor en el que se ejecuta myVirtualFrontier.
- El usuario y la contraseña de una cuenta de acceso declarada en myGateway.

- Las URLs corresondientes a las cámaras que haya en la IF.

Una vez finalizada la configuración del elemento ENLARGE, ya están implementados los canales de comunicación de la figura $2 \mathrm{y}$ es posible acceder desde el navegador a los pines que se hayan definido en el programa de control. Se indican a continuación dos ejemplos muy simples:

La orden para activar el pin 4 es:

arduinoWeb.digitalWrite(4,true);

Y para posicionar el servo a $90^{\circ}$, se usa:

arduinoWeb.servoWrite(9,90);

Para la interacción usuario-IF, la aplicación EJSS aporta un conjunto de elementos que permiten crear la vista de myUI, figura 8 . Generalmente, myUI está formada por flujos de video, gráficos de evolución de parámetros, botones, deslizadores, etc.

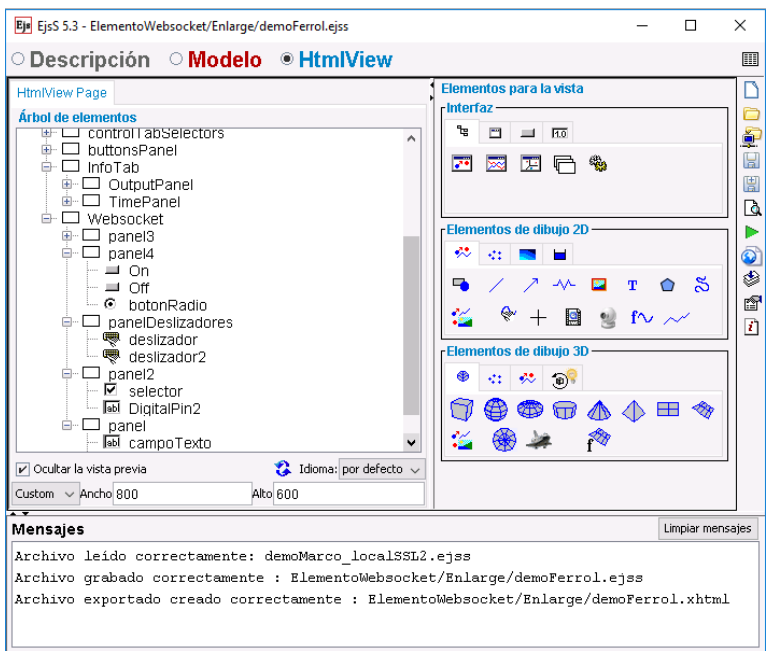

Figura 8. Elementos de EJSS para desarrollar la vista de myUI

Por ejemplo, si se necesita que un deslizador controle el servo conectado en el pin 9, se arrastará a la vista de EJSS un deslizador y se editará. Aparecerá entonces la pantalla mostrada en la figura 9 .

En el campo Value de la figura 9 se introducirá el identificador de una variable declarada $y$ en el apartado onChange hay que indicar la orden:

arduinoWeb.servoWrite(9,des2); 


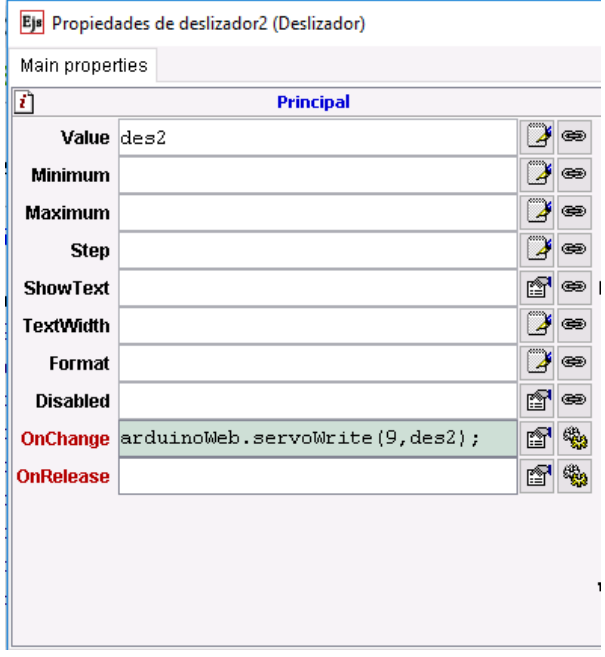

Figura 9. Vista de configuración de un deslizador en EJSS.
En cualquier fase del desarrollo de myUI se puede previsualizar el resultado pulsando el botón "Ejecutar". De esta forma, se puede comprobar en un navegador como va evolucionando myUI.

Una vez completada myUI, se selecciona la opción de empaquetarla en un fichero .zip, que será el que se suba a myGateway.

En la figura 10 se presenta myUI correspondiente a una IF ejemplo que contiene dos servomotores para mover el punto de vista del flujo de video (que se activan mediante los deslizadores que enmarcan la imagen). Además, la IF contiene un led RGB en el que se regula la intensidad luminosa, cuyo valor se representa en la gráfica de la figura 10. El led se activa y se desactiva mediante el botón On/Off.

\section{A https://agrabida.uhu.es/1006/gui/up_demoFerrol3.zip/demoFerrol3_Simulation_es.xhtml}

मे 0 m.
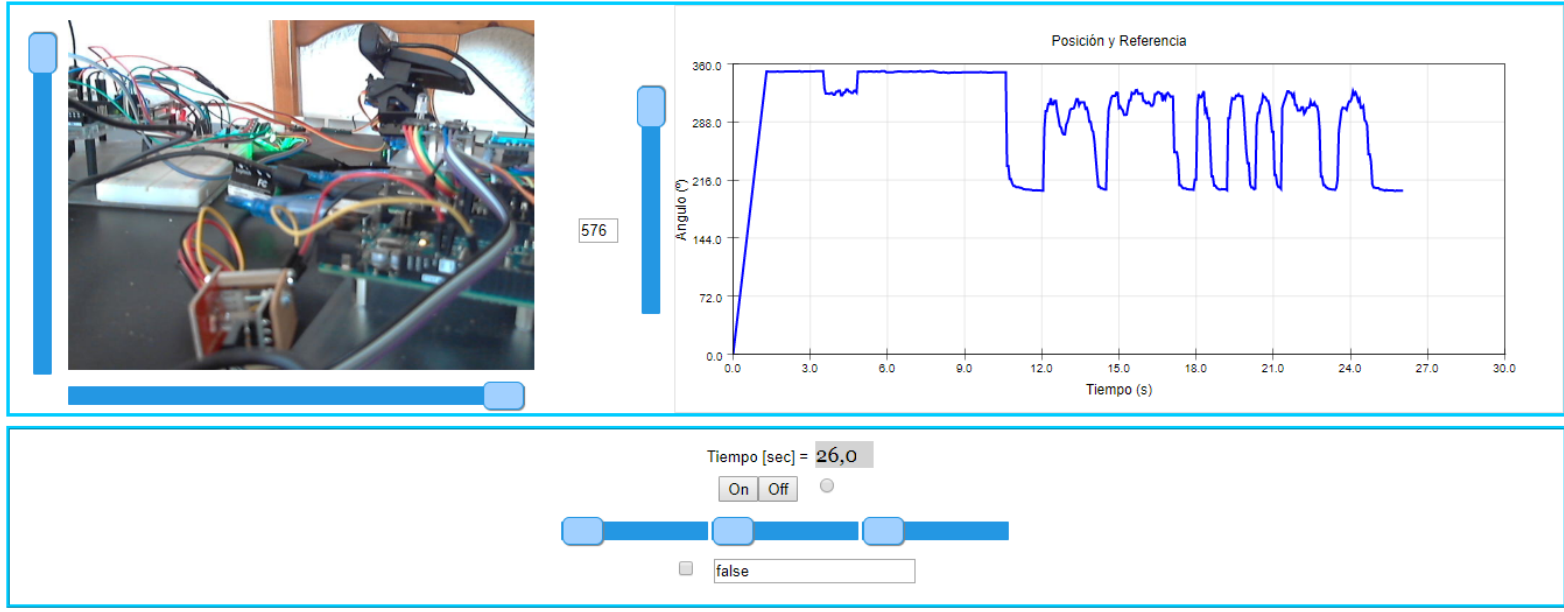

Figura 10. myUI de un IF ejemplo.

\section{CONCLUSIONES}

En este artículo se presenta un dispositivo denominado myGateway que, como parte de un sistema completo de comunicaciones denominado ENLARGE, permite ofertar como servicio en la nube el aceso desde internet a cualquier planta de laboratorio docente o de investigación, cualquier planta industrial o conuunto de dispositivos domésticos. Los accesos a la misma son seguros, controlados y ordenados mediante distintos perfiles de usuario. En artículo presenta además la forma de configurar myGateway y de desarrollar el programa de control y el de interacción del usuario con la planta, usando aplicaciones de libre distribución. Este proceso de desarrollo es sencillo y puede ser realizado por desarrolladores que no sean expertos en comunicaciones sino en el proceso industrial de que se trate.

\section{THE ADVANTAGES OF IoT IN THE DESIGN OF REMOTE LABORATORIES: myGATEWAY}

\begin{abstract}
In the time of the internet of things, the control of any household appliance through a smart device is relatively easy. With significative challenges, that revolution is reaching the industries. However, in the field of education, it still seems really complicated to make the laboratories accessible from the Internet. To achieve it, this paper presents a device that allows any didactic or research experience to be integrated
\end{abstract}


as a cloud service, whatever its nature and which uses any communication protocol between the different devices involved. Its installation and configuration are simple and intuitive, so the person who sets it up will not have to be a communications expert. The device is part of a larger system called ENLARGE.

Keywords: Engineering education, remote laboratories, internet of things, communications, cloud.

\section{Referencias}

[1] L. Atzori, A. Iera, y G. Morabito, «The Internet of Things: A survey», Comput. Netw., vol. 54, n. ${ }^{\circ} 15$, pp. 2787-2805, oct. 2010.

[2] S. Pinto, T. Gomes, J. Pereira, J. Cabral, y A. Tavares, «IIoTEED: An Enhanced, Trusted Execution Environment for Industrial IoT Edge Devices», IEEE Internet Comput., vol. 21, n. ${ }^{\circ} 1$, pp. 40-47, ene. 2017.

[3] J. Li, F. R. Yu, G. Deng, C. Luo, Z. Ming, y Q. Yan, «Industrial Internet: A Survey on the Enabling Technologies, Applications, and Challenges», IEEE Commun. Surv. Tutor., vol. 19, n. ${ }^{\circ} 3$, pp. 1504-1526, thirdquarter 2017.

[4] P. P. Ray, «A survey of IoT cloud platforms», Future Comput. Inform. J., vol. 1, n. ${ }^{0} 1$, pp. 3546, dic. 2016.

[5] I. Ruano Ruano, J. Gámez García, y J. Gómez Ortega, «Laboratorio Web SCORM de Control PID con Integración Avanzada», Rev. Iberoam. Automática E Informática Ind. RIAI, vol. 13, n. ${ }^{\circ}$ 4, pp. 472-483, oct. 2016.

[6] L. Iannucci, M. Parvis, E. D. Francia, y S. Grassini, «iHomeX: An Internet-Enabled Laboratory for Long-Term Experiment Management», IEEE Trans. Instrum. Meas., vol. 67, n. ${ }^{\circ}$ 5, pp. 1142-1149, may 2018.

[7] I. Angulo, L. Rodrìguez-Gil, y J. Garcìa-Zubìa, «Scaling up the Lab: An Adaptable and Scalable Architecture for Embedded Systems
Remote Labs», IEEE Access, vol. 6, pp. 16887-16900, 2018.

[8] A. Beghi, A. Cervato, y M. Rampazzo, «A Remote Refrigeration Laboratory for Control Engineering Education», IFAC-Pap., vol. 48, n. ${ }^{\circ} 29$, pp. 25-30, ene. 2015.

[9] «LabVIEW 2019 - National Instruments». [En línea]. Disponible en: http://www.ni.com/eses/shop/labview/labview-details.html.

[Accedido: 20-may-2019].

[10] «MathWorks - Makers of MATLAB and Simulink». [En línea]. Disponible en: https://es.mathworks.com/. [Accedido: 20may-2019].

[11] «Arduino - Home». [En línea]. Disponible en: https://www.arduino.cc/. [Accedido: 04-feb2019].

[12] «Phidgets Inc. - Products for USB Sensing and Control». [En línea]. Disponible en: https://www.phidgets.com/? [Accedido: 17abr-2019].

[13] «Orange Pi en español, la alternativa a Raspberry Pi». [En línea]. Disponible en: https://orangepiweb.es/. [Accedido: 20-may2019].

[14] «Easy Java Simulations Wiki | Main / EJS Home Page». [En línea]. Disponible en: https://www.um.es/fem/EjsWiki/?userlang=es. [Accedido: 04-feb-2019].

[15] L. de la Torre, J. Sánchez, T. F. Andrade, y M. T. Restivo, «Easy creation and deployment of Javascript remote labs with EjsS and Moodle», en 2016 13th International Conference on Remote Engineering and Virtual Instrumentation (REV), 2016, pp. 260-261.

C 2019 by the authors. Submitted for possible open access publication under the terms and conditions of the Creative Commons Attribution CC BY-NC-SA 4.0 license (https://creativecommons.org/licenses/bync-sa/4.0/deed.es).

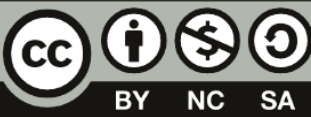

